\title{
Efficient Relational Storage and Retrieval of XML Documents
}

\author{
Albrecht Schmidt Martin Kersten Menzo Windhouwer Florian Waas \\ CWI \\ Kruislaan 413 \\ P.O. Box 94079 \\ 1090 GB Amsterdam \\ The Netherlands \\ firstname.lastname@cwi.nl
}

\begin{abstract}
In this paper, we present a data and an execution model that allow for efficient storage and retrieval of XML documents in a relational database. The data model is strictly based on the notion of binary associations: by decomposing XML documents into small, flexible and semantically homogeneous units we are able to exploit the performance potential of vertical fragmentation. Moreover, our approach provides clear and intuitive semantics, which facilitates the definition of a declarative query algebra. Our experimental results with large collections of XML documents demonstrate the effectiveness of the techniques proposed.
\end{abstract}

\section{INTRODUCTION}

XML increasingly assumes the role of the de facto standard data exchange format in Web database environments. Modeling issues that arise from the discrepancy between semi-structured data on the one hand side and fully structured database schemas on the other have received special attention. Database researchers provided valuable insights to bring these two areas together. The solutions proposed include not only XML domain specific developments but also techniques that build on object-oriented and relational database technology (e.g., see [7, 1, 11, 15, 8, 16, 10, 2, 12]).

To make XML the language of Web databases, performance issues are the upcoming challenge that has to be met. Database support for XML processing can only find the widespread use that researchers anticipate if storage and retrieval of documents satisfy the demands of impatient surfers.

In this paper, we are concerned with providing effective tools for the management of XML documents. This includes tight interaction between established standards on the declarative conceptual level like the DOM [18] and efficient physical query execution. Starting from the syntax tree representation of a document, we propose a data model that is based on a complete binary fragmentation of the document tree. This way, all relevant associations within a document like parentchild relationships, attributes, or topological orders can be intuitively described, stored and queried. In contrast to general graph databases like Lore [1], we draw benefit from the basic tree structure of the document and incorporate information about the association's position within the syntax tree relative to the root into our data model. References such as IDREFs that escape the tree structure are taken care of by views on the tree structure. Associations that provide semantically related information are stored together in the binary relations of the database repository. Along with the decomposition schema we also present a method to translate queries formulated on paths of the syntax tree into expressions of an algebra for vertically fragmented schemas [4].

Our approach is distinguished by two features. Firstly, the decomposition method is independent of the presence of DTDs, but rather explores the structure of the document at parse time. Information on the schema is automatically available after the decomposition. Secondly, it reduces the volume of data irrelevant to a query that has to be processed during querying. Storing associations according to their context in the syntax tree provides tables that contain semantically closely related information. As a result, data relevant for a given query can be accessed directly in form of a separate table avoiding large and expensive scans over irrelevant data.

Reservations exist that a high degree of fragmentation might incur increased efforts to reconstruct the original document, or parts of it. However, as our quantitative assessment shows, the number of additional joins is fully made up for as they involve only little data volume. Our approach displays distinctly superior performance compared to previous work.

\section{DATA MODEL AND ALGEBRA}

XML documents are commonly represented as syntax trees. With string and int denoting sets of character strings and integers and oid being the set of unique object identifiers, we can define a XML document formally (e.g., see [19]):

Definition 1. An XML document is a rooted tree $d=$ $\left(V, E, r\right.$, label $_{E}$, label $_{A}$, rank $)$ with nodes $V$ and edges $E \subseteq$ $V \times V$ and a distinguished node $r \in V$, the root node. The function label $_{E}: V \rightarrow$ string assigns labels to nodes, i.e., elements; label $_{A}: V \rightarrow$ string $\rightarrow$ string assigns pairs of strings, attributes and their values, to nodes. Character Data (CDATA) are modeled as a special 'string' attribute of cdata nodes, rank $: V \rightarrow$ int establishes a ranking to allow for an order among nodes with the same parent node. For 


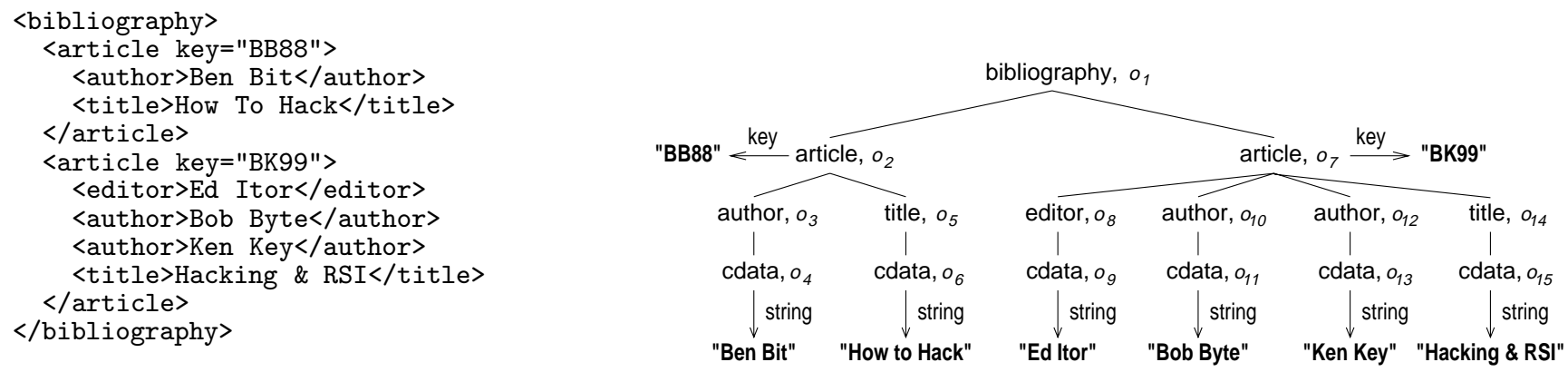

Figure 1: XML document and corresponding syntax tree

elements without any attributes label $_{A}$ maps to the empty set.

Figure 1 shows an XML document, which describes a fragment of a bibliography, alongside its associated syntax tree. The representation is largely self-explanatory, $o_{i}$ denote object identifiers (OIDs) whose assignment is arbitrary, e.g., depthfirst traversal order. We apply the common simplification not to differentiate between PCDATA and CDATA nor do we take rich datatypes into account.

\subsection{Preliminaries}

Before we discuss techniques how to store a syntax graph as a database instance, we introduce the concepts of associations and path summaries. They identify spots of interest and constitute the basis for the Monet XML Model. ${ }^{1}$

Definition 2. A pair $(o, \cdot) \in$ oid $\times($ oid $\cup$ int $\cup$ string $)$ is called an association.

The different types of associations describe different parts of the tree: associations of type oid $\times$ oid represent edges, i.e., parent-child relationships. Attribute values (including character data, represented by vertices with label 'string', that start from 'cdata' labelled nodes) are modeled by associations of type oid $\times$ string, while associations of type oid $\times$ int are used to preserve the topology of a document.

DeFinition 3. For a node o in the syntax tree, we denote the sequence of labels along the path (vertex and edge labels) from the root to o with $\operatorname{path}(o)$.

As an example, consider the node with OID $o_{3}$ in Figure 1; its path is bibliography $\stackrel{e}{\rightarrow}$ article $\stackrel{e}{\rightarrow}$ author. The corresponding character data string "Ben Bit" has path bibliography $\stackrel{e}{\rightarrow}$ article $\stackrel{e}{\rightarrow}$ author $\stackrel{e}{\rightarrow}$ cdata $\stackrel{a}{\rightarrow}$ string, where $\stackrel{e}{\rightarrow}$ denotes edges to elements and $\stackrel{a}{\rightarrow}$ to attributes.

Paths describe the position of the element in the graph relative to the root node and we use path(o) to denote the type of the association $(\cdot, o)$. The set of all paths in a document is called the document's path summary.

\footnotetext{
${ }^{1}$ We chose the name Monet XML Model because the homegrown database engine Monet [4] serves as implementation platform.
}

\subsection{The Monet XML Model}

As we pointed out at the beginning, the question central to querying XML documents is how to store the syntax tree as a database instance that provides efficient retrieval capabilities. Given Definition 1 the tree could be stored using a single database table for the parent-child relations (similar to [17]), another one for the elements labels and so on. Though space effective, such a decomposition makes querying expensive by enforcing scans over large amounts of data irrelevant to a query, since structurally unrelated data are stored in the same tables. Even if the query consist of a few joins only, large data volumes may have to be processed (see [10] for a discussion of storage schemes of this kind).

We pursue a rather different approach using the structures defined above, i.e., storing all associations of the same type in the same binary relation. A relation that contains the tuple $(\cdot, o)$ is named path $(o)$, and, conversely, a tuple is stored in exactly one relation.

Definition 4. Given an XML document $d$, the Monet transform is a quadruple $M_{t}(d)=(r, \mathbf{R}, \mathbf{A}, \mathbf{T})$ where

$\mathbf{R}$ is the set of binary relations that contain all associations between nodes;

A is the set of binary relations that contain all associations between nodes and their attribute values, including character data;

$\mathbf{T}$ is the set of binary relations that contain all pairs of nodes and their rank;

$r$ remains the root of the document.

Encoding the path to a component into the name of the relation often achieves a significantly higher degree of fragmentation than implied by plain data guides [11]. In other words, we use path to group semantically related associations into the same relation. As a direct consequence of the decomposition schema, we do not need to introduce novel features on the storage level to cope with irregularities induced by the semi-structured nature of XML, which are typically taken care of by NULLs or overflow tables [8]. Moreover, it should be noted, that the complete decomposition is linear in the size of the document with respect to running 


$$
\begin{aligned}
& \text { bibliography } \stackrel{e}{\rightarrow} \text { article }=\left\{\left\langle o_{1}, o_{2}\right\rangle,\left\langle o_{1}, o_{7}\right\rangle\right\}, \\
& \text { bibliography } \stackrel{e}{\rightarrow} \text { article } \stackrel{e}{\rightarrow} \text { author }=\left\{\left\langle o_{2}, o_{3}\right\rangle,\left\langle o_{7}, o_{10}\right\rangle,\left\langle o_{7}, o_{12}\right\rangle\right\} \\
& \text { bibliography } \stackrel{e}{\rightarrow} \text { article } \stackrel{e}{\rightarrow} \text { author } \stackrel{e}{\rightarrow} \text { cdata }=\left\{\left\langle o_{3}, o_{4}\right\rangle,\left\langle o_{10}, o_{11}\right\rangle,\left\langle o_{12}, o_{13}\right\rangle\right\} \text {, } \\
& \text { bibliography } \stackrel{e}{\rightarrow} \text { article } \stackrel{e}{\rightarrow} \text { author } \stackrel{e}{\rightarrow} \text { cdata } \stackrel{a}{\rightarrow} \text { string }=\left\{\left\langle o_{4}, \text { "Ben Bit" }\right\rangle,\left\langle o_{11} \text {, "Bob Byte" }\right\rangle,\left\langle o_{13}, \text { "Ken Key" }\right\},\right. \\
& \text { bibliography } \stackrel{e}{\rightarrow} \text { article } \stackrel{e}{\rightarrow} \text { title }=\left\{\left\langle o_{2}, o_{5}\right\rangle,\left\langle o_{7}, o_{14}\right\rangle\right\}, \\
& \text { bibliography } \stackrel{e}{\rightarrow} \text { article } \stackrel{e}{\rightarrow} \text { title } \stackrel{e}{\rightarrow} \text { cdata }=\left\{\left\langle o_{5}, o_{6}\right\rangle,\left\langle o_{14}, o_{15}\right\rangle\right\}, \\
& \text { bibliography } \stackrel{e}{\rightarrow} \text { article } \stackrel{e}{\rightarrow} \text { title } \stackrel{e}{\rightarrow} \text { cdata } \stackrel{a}{\rightarrow} \text { string }=\left\{\left\langle o_{6}, \text { "How to Hack" }\right\rangle,\left\langle o_{15}, \text { "Hacking \& RSI" }\right\rangle\right\}, \\
& \text { bibliography } \stackrel{e}{\rightarrow} \text { article } \stackrel{e}{\rightarrow} \text { editor }=\left\{\left\langle o_{7}, o_{8}\right\rangle\right\}, \\
& \text { bibliography } \stackrel{e}{\rightarrow} \text { article } \stackrel{e}{\rightarrow} \text { editor } \stackrel{e}{\rightarrow} \text { cdata }=\left\{\left\langle o_{8}, o_{9}\right\rangle\right\}, \\
& \text { bibliography } \stackrel{e}{\rightarrow} \text { article } \stackrel{e}{\rightarrow} \text { editor } \stackrel{e}{\rightarrow} \text { cdata } \stackrel{a}{\rightarrow} \text { string }=\left\{\left\langle o_{9}, \text { "Ed Itor" }\right\rangle\right\}, \\
& \text { bibliography } \left.\stackrel{e}{\rightarrow} \text { article } \stackrel{a}{\rightarrow} \text { key }=\left\{\left\langle o_{2}, " \mathrm{BB} 88 "\right\rangle,\left\langle o_{7}, \text { "BK99" }\right\rangle\right\}\right\}
\end{aligned}
$$

\section{Figure 2: Monet transform $M_{t}$ of the example document}

time. Concerning memory requirements, it is in $O(h), h$ being the height of the syntax tree, in addition to the space the binary relations in the database engine occupy, i.e., it is not necessary to materialize the complete syntax tree.

Proposition 1. The above mapping is lossless, i.e., for an XML document $d$ there exists an inverse mapping $M_{t}^{-1}$ such that $d$ and $M_{t}^{-1}\left(M_{t}(d)\right)$ are isomorphic.

A sketch of the proof of Proposition 1 is given in the appendix. Figure 2 shows the Monet transform of the example document.

The Monet transform also enables an object-oriented perspective, i.e., object being interpreted as node in the syntax tree, which is often more intuitive to the user and is adopted by standards like the DOM [18]. Particularly in querying, approaches that bear strong similarities with object-oriented techniques have emerged. Given the Monet transform, we have the necessary tools at hand to reconcile the relational perspective with the object-oriented view.

It is natural to re-assemble an object with OID $o$ from those associations whose first component is $o$ : e.g., the object $\operatorname{object}\left(o_{2}\right)=\left\{k e y\left\langle o_{2}, " \mathrm{BB} 88 "\right\rangle\right.$, author $\left\langle o_{2}, o_{3}\right\rangle$, title $\left.\left\langle o_{2}, o_{5}\right\rangle\right\}$ is easily converted into an instance of a suitably defined class article with members key, author and title. However, XML is regarded as an incarnation of the semi-structured paradigm. One consequence of this is that we cannot expect all instances of one type to share the same structure. In the example, the second publication does have an editor element whereas the first does not. We therefore distinguish between two kinds of associations: (strong) associations and weak associations. Strong associations constitute the structured part of XML - they are present in every instance of a type; weak associations account for the semi-structured part: they may or may not appear in a given instance. Objects $O_{2}$ and $o_{7}$ reflect this: $o_{7}$ has a editor member whereas $\mathrm{O}_{2}$ has not. Therefore, we define the following:
Definition 5. An object o is a set of strong and weak associations $\left\{A_{1}\left\langle o, o_{1}\right\rangle, A_{2}\left\langle o, o_{2}\right\rangle, \ldots\right\}$.

The next question we address directly arises from the modeling of objects: How can we re-formulate queries from an object-oriented setting to queries in relational Monet XML?

\subsection{Execution Model and Algebra}

The unified view provided by the Monet XML model extends directly to querying. For the relational layer, a multitude of operators implementing the relational algebra, including specialties intrinsic to vertical fragmented schemas, have been proposed. Hence, we omit a discussion of technical issues concerning bare, relational query processing in the context of vertical fragmentation and refer the interested reader to [4] for a comprehensive overview.

More interesting is the actual translation of an OQL-like query to match the facilities of the underlying query execution engine. We only outline the translation by an example query. The process bears strong resemblance to mapping techniques developed to implement object-oriented query interfaces on relational databases; thus, we can resort to the wealth of techniques developed in that field. See [5] for a comparative analysis of different query languages for XML.

Consider the following query which selects those of Ben Bit's publications whose titles contain the word 'Hack'; the semantics of the statements are similar to [3]:

$$
\begin{array}{ll}
\text { select } & p \\
\text { from } & \text { bibliography } \stackrel{e}{\rightarrow} \text { article } p \\
& p \stackrel{e}{\rightarrow} \text { author } \stackrel{e}{\rightarrow} \text { cdata } a \\
& p \stackrel{e}{\rightarrow} \text { title } \stackrel{e}{\rightarrow} \text { cdata } t \\
\text { where } & a=\text { "Ben Bit" and } t \text { like "Hack"; }
\end{array}
$$

The query consists of two blocks, a specification of the elements involved, which translates to computing the proper binary relations, and constraints that define the actual processing. For resolving path expressions, we need to distin- 
guish two types of variables in the from clause: variables that specify sets, $p$ in the example, and variables, which specify associations, $a$ and $t$.

We collapse each path expression that is not available in the database by joining the binary relations along the path specification. This establishes an association between the first and last element of the path. Finally, we take the intersection of the specified elements. Matching the variables against the running example, the from clause specifies the following elements:

$$
\begin{aligned}
& p=\{\left.o_{2}, o_{7}\right\}, \\
& \operatorname{assoc}(p \rightarrow a)=\left\{\left(o_{2}, \text { "Ben Bit" }\right),\left(o_{7}, \text { "Bob Byte" }\right),\right.\left.\left(o_{7}, \text { "Ken Key" }\right)\right\}, \\
& \operatorname{assoc}(p \rightarrow t)=\left\{\left(o_{2}, \text { "How To Hack" }\right),\right. \\
&\left.\left(o_{7}, \text { "Hacking \& RSI" }\right)\right\}
\end{aligned}
$$

Queries containing regular expressions over paths directly benefit from the availability of the path summary. Standard methods for the evaluation of regular expressions can be applied to the textual representation of the paths and enable the immediate selection of the candidate relations.

The evaluation of the where clause is not of particular interest in this context. Though processing of binary tables differs from the conventional relational model in several aspects, these differences have no direct impact on our method.

\section{QUANTITATIVE ASSESSMENT}

We assess the techniques proposed with respect to size of the resulting database, as well as querying and browsing the database. As application domains we chose readily available XML document collection: the ACM SIGMOD Anthology [13], Webster's Dictionary [9], and Shakespeare's Plays [6].

We implemented Monet XML within the Monet database server [4]. The measurements were carried out on an Silicon Graphics 1400 Server with 1 GB main memory, running at $550 \mathrm{MHz}$. For comparisons with related work, we used a Sun UltraSPARC-IIi with $360 \mathrm{MHz}$ clock speed and $256 \mathrm{MB}$ main memory.

Database Size. The resulting sizes of the decomposition scheme are a critical issue. Theoretically, the size of the path summary can be linear in the size of the document as the worst case - if the document is completely un-structured. However, in practical applications, we typically find large structured portions within each document. Table 1 shows the database sizes for our examples in comparison with the size of the original XML code. The third column contains the number of tables, i.e., the size of the path summary. The last column shows the complete time needed to parse, decompose and store the documents.

It leaps out that the Monet XML version of the ACM Anthology is of smaller size than the original document. This reduction is due to the 'automatic' compression inherent in the Monet transform (tag names are stored only once as metainformation) and the removal of redundantly occurring character data. For example there are only few different publishers compared to the number of entries in general. In

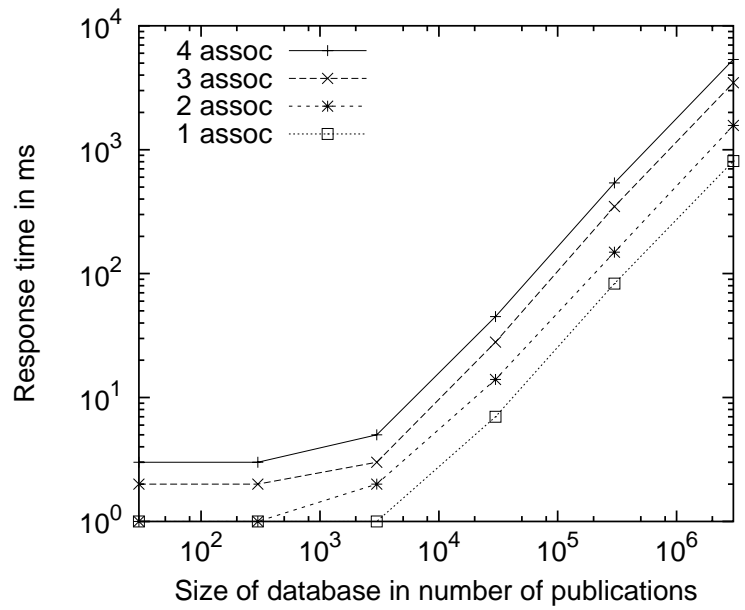

Figure 3: Scaling of document

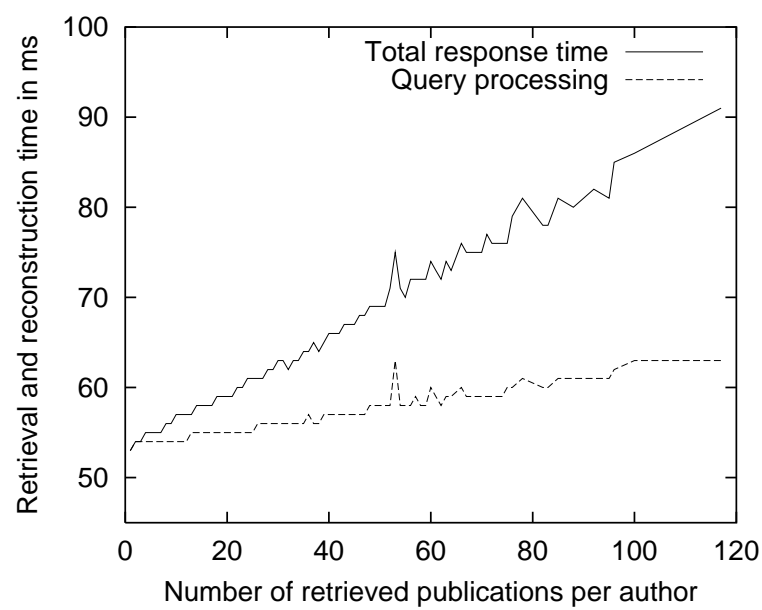

Figure 4: Response time vs. result size

the decomposition, full entries of these fields can be replaced with references; this is done automatically by the DBMS. We can expect similar effects to occur with other decomposition schemas, like object-oriented mappings.

Scaling. In order to inspect the scaling behavior of our technique we varied the size of the underlying document. In doing so, we took care to maintain the ratio of different elements and attributes of the original document. We scaled the ACM Anthology from 30 to $3 \cdot 10^{6}$ publications which corresponds to XML source size between $10 \mathrm{~KB}$ and $1 \mathrm{~GB}$. Size of the database and time scaled linear in the size of the XML document.

Querying. To test for query performance under scaling we ran 4 queries consisting of path expressions of length 1 through 4 for various sizes of the Anthology. As Figure 3 shows, the response times for each query, given as a function of the size of the document, is linear in the size of the database. Only for small sizes of the database, the response time is dominated by the overhead of the database system. Notice, both axes are logarithmic. 


\begin{tabular}{|l|r|r|r|r|}
\hline Documents & size in XML & size in Monet XML & \#Tables & Loading \\
\hline \hline ACM Anthology & $46.6 \mathrm{MB}$ & $44.2 \mathrm{MB}$ & 187 & $30.4 \mathrm{~s}$ \\
\hline Shakespeare's Plays & $7.9 \mathrm{MB}$ & $8.2 \mathrm{MB}$ & 95 & $4.5 \mathrm{~s}$ \\
\hline Webster's Dictionary & $56.1 \mathrm{MB}$ & $95.6 \mathrm{MB}$ & 2587 & $56.6 \mathrm{~s}$ \\
\hline
\end{tabular}

Table 1: Sizes of document collections in XML and Monet XML format

\begin{tabular}{|c|c|r|r|r|r|r|r|r|r|r|r|}
\hline & & Q1 & Q2 & Q3 & Q4 & Q5 & Q6 & Q7 & Q8 & Q9 & Q10 \\
\hline \hline 1A & Monet XML & 1.2 & 5.6 & 6.8 & 8.0 & 4.4 & 4.9 & 5.0 & 5.0 & 8.8 & 12.7 \\
\hline 2A & SYU / Postgres & 150 & 180 & 160 & 180 & 190 & 340 & 350 & 370 & 1300 & 1040 \\
\hline \hline 1B & Monet XML & - & 4.4 & 5.6 & 6.8 & 3.2 & 3.7 & 3.8 & 3.8 & 7.6 & 11.5 \\
\hline 2B & SYU / Postgres & - & 30 & 10 & 30 & 40 & 190 & 200 & 220 & 1150 & 890 \\
\hline
\end{tabular}

Table 2: Comparison of response times for query set of SYU

Only few of the performance analyses published so far offer the possibility to reproduce and compare results, which makes meaningful comparison difficult at this time. The results we use to compare Monet XML against were reported in [16] who implemented their algorithms as a front-end to Postgres. In [16], the authors propose a set of 10 queries using Shakespeare's plays [6] as an application domain. We refer to their approach as SYU in the following. In Table 2 we contrasted response times of Monet XML with SYU obtained from experiments on the abovementioned Sun Workstation.

The figures display a substantial difference in response time showing that Monet XML outruns the competitor by up to two orders of magnitude (rows $1 \mathrm{~A}, 2 \mathrm{~A}$ ). The times for SYU include a translation of XQL to SQL that is handled outside the database server. To allow for this difference, we additionally computed the response times relative to query 1 for both systems separately, assuming that preprocessing costs have a constant contribution. These figures exhibit actual query processing time only (rows $1 \mathrm{~B}, 2 \mathrm{~B}$ ). Monet XML shows an increase of processing time by less than $12 \mathrm{~ms}$ whereas $\mathrm{SYU}$ is up to $1150 \mathrm{~ms}$ slower than its fastest response time.

An analysis of the figures exhibits the advantages of the Monet model. While SYU store basically all data on a single heap and have to scan these data repeatedly, the Monet transform yields substantially smaller data volumes. In some extreme cases, the query result is directly available in Monet XML without any processing and only needs to be traversed and output. Another noticeable difference concerns the complexity of queries: the straight-forward semantics of the Monet XML model result in relatively simple queries; conversely, the compiled SQL statements that SYU present are quite complex.

The comparison with Lore [14] exhibited essentially the same trends on small document instances. However, we were not able to bulkload and query larger documents like the ACM anthology as Lore requested more than the available $1 \mathrm{~GB}$ main memory. In contrast, using Monet XML we engineered a system functionally equivalent to the online DBLP server [13] that operated in less than $130 \mathrm{MB}$.

Browsing a database. Our last experiments aim at assessing the systems capabilities with respect to browsing. As an example consider a typical query as it is run on the Anthology server several thousand times a day: Retrieve all conference publications for a given author. Clearly, the size of the output may vary drastically and it is of particular interest for a browsing session that response times are kept low independent of the size of the answer.

Figure 4 shows both the total response time including textual rendering and response time of the repository. As expected, the time for rendering the output increases significantly yet linear in the result size. However, the response time of the repository increases at significantly lower rate. This is due to the reconstruction of the associations in form of joins rather than chasing individual chains of pointers. Even for authors with a large number of publications the overall response time is well under one tenth of a second, which makes interactive browsing affordable. Also note that the lower line in Figure 4 could also be interpreted as the cost of constructing a view while the upper line additionally includes rendering the view to textual XML.

The results presented demonstrate the performance potential of our approach deploying fully vertical fragmentation. As the low response times show, reducing the data volume involved in single database operations on the expense of additional joins pays very well not only in terms of overall performance but also when scaling is an issue.

\section{CONCLUSIONS}

We presented a data model for efficient processing of XML documents. Our experiences show that it is worth taking the plunge and fully decompose XML documents into binary associations. The experimental results obtained with a prototype implementation based on Monet underline the viability of our approach: the effort to reduce data volume quickly pays off as gains in efficiency. Overall, our approach combines the elegance of clear semantics with a highly efficient execution model by means of a simple and effective mapping between XML documents and a relational schema.

Concerning future work, we will concentrate on exploring possibilities of parallel processing and efficient handling of multi-query workloads as found in typical interactive Webbased information systems. As we have seen with own experiments, there is also the need for a general, standardized methodology that allows conclusive performance analyses and facilitates comparisons of different approaches. 


\section{REFERENCES}

[1] S. Abiteboul, D. Quass, J. McHugh, J. Widom, and J. L. Wiener. The Lorel Query Language for Semistructured Data. International Journal on Digital Libraries, 1(1):68-88, 1997.

[2] S. AG. Tamino - Technical Description. available at http://www. softwareag.com/tamino/technical/ description.htm.

[3] C. Beeri and Y. Tzaban. SAL: An Algebra for Semistructured Data and XML. In International Workshop on the Web and Databases, pages 37-42, Pennsylvania, USA, 1999.

[4] P. A. Boncz and M. L. Kersten. MIL Primitives for Querying a Fragmented World. The VLDB Journal, 8(2):101-119, 1999.

[5] A. Bonifati and S. Ceri. Comparative Analysis of Five XML Query Languages. ACM SIGMOD Record, 1(29), 2000.

[6] J. Bosak. Sample XML documents. shakespeare.1.01.xml.zip available at ftp:// sunsite.unc.edu/pub/sun-info/standards/xml/eg/.

[7] P. Buneman, S. B. Davidson, G. G. Hillebrand, and D. Suciu. A Query Language and Optimization Techniques for Unstructured Data. In Proc. of the ACM SIGMOD Int'l. Conf. on Management of Data, pages 505-516, Montreal, Canada, 1996.

[8] A. Deutsch, M. F. Fernandez, and D. Suciu. Storing Semistructured Data with STORED. In Proc. of the ACM SIGMOD Int'l. Conf. on Management of Data, pages 431-442, Philadephia, PA, USA, 1999.

[9] M. Dyck. The GNU version of The Collaborative International Dictionary of English, presented in the Extensible Markup Language. available at http://metalab.unc.edu/webster/.

[10] D. Florescu and D. Kossmann. Storing and Querying XML Data Using an RDBMS. Data Engineering Bulletin, 22(3), 1999.

[11] R. Goldman and J. Widom. Dataguides: Enabling Query Formulation and Optimization in Semistructured Databases. In Proc. of the Int'l. Conf. on Very Large Data Bases, pages 436-445, Athens, Greece, 1997.

[12] C. Kanne and G. Moerkotte. Efficient Storage of XML Data. In Proceedings of the 16th International Conference on Data Engineering, page 198, 2000.

[13] M. Ley. DBLP Bibliography. http: //www. informatik. uni-trier.de:8000/ ley/db/.

[14] J. McHugh, S. Abiteboul, R. Goldman, D. Quass, and J. Widom. Lore: A Database Management System for Semistructured Data. ACM SIGMOD Record, 3(26), 1997.
[15] J. Shanmugasundaram, K. Tufte, G. He, C. Zhang, D. DeWitt, and J. Naughton. Relational Databases for Querying XML Documents: Limitations and Opportunities. In Proc. of the Int'l. Conf. on Very Large Data Bases, pages 302-314, Edinburgh, UK, 1999.

[16] T. Shimura, M. Yoshikawa, and S. Uemura. Storage and Retrieval of XML Documents Using Object-Relational Databases. In Database and Expert Systems Applications, pages 206-217. Springer, 1999.

[17] R. van Zwol, P. Apers, and A. Wilschutz. Implementing semi-structured data with MOA. In Workshop on Query Processing for Semistructured data and Non-Standard Data Formats (in conjunction with ICDT), 1999.

[18] W3C. Document Object Model (DOM). available at http://www.w3.org/DOM/.

[19] W3C. Extensible Markup Language (XML) 1.0. available at http://www.w3.org/TR/1998/REC-xml-19980210.

\section{APPENDIX}

Proof of Proposition 1. Definition 4 introduces the Monet transform $M_{t}(d)=(r, \mathbf{R}, \mathbf{A}, \mathbf{T})$ of a document $d$. For a document $d$ the sets $\mathbf{R}, \mathbf{A}$ and $\mathbf{T}$ are computed as follows: for elements:

$$
\mathbf{R}=\bigcup_{\left(o_{i}, o_{j}, s\right) \in \tilde{E}}\left[\operatorname{path}\left(o_{i}\right) \stackrel{e}{\rightarrow} s\right]\left\langle o_{i}, o_{j}\right\rangle
$$

for attributes including CDATA:

$$
\begin{aligned}
\mathbf{A}= & \bigcup_{\left(o_{i}, s_{1}, s_{2}\right) \in \text { label }_{A}}\left[\operatorname{path}\left(o_{i}\right) \stackrel{a}{\rightarrow} s_{1}\right]\left\langle o_{i}, s_{2}\right\rangle, \\
& \text { for ranking integers: } \\
\mathbf{T}= & \left.\bigcup_{\left(o_{i}, i\right) \in \text { rank }}\left[\text { path }\left(o_{i}\right) \rightarrow \text { rank }\right]\left\langle o_{i}, i\right\rangle\right),
\end{aligned}
$$

where $E$ and label $_{E}$ are combined into one set

$$
\tilde{E}=\left\{\left(o_{1}, o_{2}, s\right) \mid\left(o_{1}, o_{2}\right) \in E, s=\operatorname{label}_{E}\left(o_{2}\right)\right\},
$$

label $_{A}$ is interpreted as a set $\subseteq$ oid $\times$ string $\times$ string as well as $r a n k \subseteq$ oid $\times$ int, and $[\exp r]$ means that the value of expr is a relation name. To see that the mapping given in definition 4 is lossless we give the inverse mapping. Given an instance of the Monet XML model $M_{t}(d)$ we can reconstruct the original rooted tree $d=\left(V, E, r\right.$, label $_{E}$, label $_{A}$, rank $)$ in the following way ( $\operatorname{second-last}(p)$ returns the second-last component of path $p$ ).

1. $V=\left\{o_{i} \mid(\exists R \in \mathbf{R})\left(\exists o_{j} \in\right.\right.$ oid $\left.): R\left\langle o_{i}, o_{j}\right\rangle\right\}$,

2. $E=\left\{\left(o_{i}, o_{j}\right) \mid(\exists R \in \mathbf{R}): R\left\langle o_{i}, o_{j}\right\rangle\right\}$,

3. $r$ remains,

4. label $_{E}=\left\{\left(o_{i}, s\right) \mid(\exists R \in \mathbf{R})\left(\exists o_{j} \in\right.\right.$ oid $)(\exists s \in$ string $)$ : $\left.R\left\langle o_{i}, o_{j}\right\rangle \wedge \operatorname{second-last}(R)=s\right\}$,

5. label $_{A}=\left\{\left(o_{i}, s_{1}, s_{2}\right) \mid(\exists A \in \mathbf{A}): A\left\langle o_{i}, s_{2}\right\rangle \wedge \operatorname{last}(A)=\right.$ $\left.s_{1}\right\}$,

6. $\operatorname{rank}=\{(o, i) \mid(\exists T \in \mathbf{T}): T\langle o, i\rangle\}$. 